\title{
An integrated text mining framework for metabolic interaction network reconstruction
}

Preecha Patumcharoenpol, Narumol Doungpan, Asawin Meechai, Bairong Shen, Jonathan H Chan, Wanwipa Vongsangnak

Text mining (TM) in the field of biology is fast becoming a routine analysis for the extraction and curation of biological entities (e.g., genes, proteins, simple chemicals) as well as their relationships. Due to the wide applicability of TM in situations involving complex relationships, it is valuable to apply TM to the extraction of metabolic interactions (i.e., enzyme and metabolite interactions) through metabolic events. Here we present an integrated TM framework containing two modules for the extraction of metabolic events (Metabolic Event Extraction module - MEE) and for the construction of a metabolic interaction network (Metabolic Interaction Network Reconstruction module - MINR). The proposed integrated TM framework performed well based on standard measures of recall, precision and F-score. Evaluation of the MEE module using the constructed Metabolic Entities (ME) corpus yielded F-scores of $59.15 \%$ and $48.59 \%$ for the detection of metabolic events for production and consumption, respectively. As for the testing of the entity tagger for Gene and Protein (GP) and metabolite with the test corpus, the obtained F-score was greater than $80 \%$ for the Superpathway of leucine, valine, and isoleucine biosynthesis. Mapping of enzyme and metabolite interactions through network reconstruction showed a fair performance for the MINR module on the test corpus with F-score $>70 \%$. Finally, an application of our integrated TM framework on a big-scale data (i.e., EcoCyc extraction data) for reconstructing a metabolic interaction network showed reasonable precisions at $69.93 \%, 70.63 \%$ and $46.71 \%$ for enzyme, metabolite and enzyme-metabolite interaction, respectively. This study presents the first open-source integrated TM framework for reconstructing a metabolic interaction network. This framework can be a powerful tool that helps biologists to extract metabolic events for further reconstruction of a metabolic interaction network. The ME corpus, test corpus, source code, and virtual machine image with pre-configured software are available at www.sbi.kmutt.ac.th/ preecha/metrecon. 


\section{An integrated text mining framework for metabolic 2 interaction network reconstruction}

4 Preecha Patumcharoenpol ${ }^{1,2, *}$, Narumol Doungpan ${ }^{3}$, Asawin Meechai ${ }^{1,4}$, Bairong Shen ${ }^{2}$, Jonathan

5 H. Chan ${ }^{1,3, \S}$, Wanwipa Vongsangnak ${ }^{2,5^{*}, \S}$

$6{ }^{1}$ Systems Biology and Bioinformatics Laboratory, King Mongkut's University of Technology

7 Thonburi, Bangkok 10140, Thailand

$8{ }^{2}$ Center for Systems Biology, Soochow University, Suzhou 215006, China

$9{ }^{3}$ School of Information Technology, King Mongkut's University of Technology Thonburi,

10 Bangkok 10140, Thailand

$11{ }^{4}$ Department of Chemical Engineering, Faculty of Engineering, King Mongkut's University of

12 Technology Thonburi, Bangkok 10140, Thailand

$13{ }^{5}$ Department of Zoology, Faculty of Science, Kasetsart University, Bangkok 10900, Thailand

$15 *$ These authors contributed equally to this work

16 ¿Corresponding Authors:

17 Jonathan H. Chan ${ }^{3}$

18126 Pracha Uthit Rd, Bang Mod, Thung Khru, Bangkok 10140, Thailand

19 E-mail address: jonathan@sit.kmutt.ac.th

20 Wanwipa Vongsangnak ${ }^{5}$

2150 Ngam Wong Wan Rd, Ladyaow, Chatuchak, Bangkok 10900, Thailand

22 E-mail address: wanwipa.v@ku.ac.th 


\section{Abstract}

\section{Background}

25 Text mining (TM) in the field of biology is fast becoming a routine analysis for the extraction

26 and curation of biological entities (e.g., genes, proteins, simple chemicals) as well as their

27 relationships. Due to the wide applicability of TM in situations involving complex relationships,

28 it is valuable to apply TM to the extraction of metabolic interactions (i.e., enzyme and metabolite

29 interactions) through metabolic events. Here we present an integrated TM framework containing

30 two modules for the extraction of metabolic events (Metabolic Event Extraction module - MEE)

31 and for the construction of a metabolic interaction network (Metabolic Interaction Network

32 Reconstruction module - MINR).

\section{Results}

34 The proposed integrated TM framework performed well based on standard measures of recall, 35 precision and F-score. Evaluation of the MEE module using the constructed Metabolic Entities (ME) corpus yielded F-scores of $59.15 \%$ and $48.59 \%$ for the detection of metabolic events for

37 production and consumption, respectively. As for the testing of the entity tagger for Gene and Protein (GP) and metabolite with the test corpus, the obtained F-score was greater than $80 \%$ for the Superpathway of leucine, valine, and isoleucine biosynthesis. Mapping of enzyme and metabolite interactions through network reconstruction showed a fair performance for the MINR module on the test corpus with F-score $>70 \%$. Finally, an application of our integrated TM framework on a big-scale data (i.e., EcoCyc extraction data) for reconstructing a metabolic interaction network showed reasonable precisions at $69.93 \%, 70.63 \%$ and $46.71 \%$ for enzyme, metabolite and enzyme-metabolite interaction, respectively. 


\section{Conclusions}

48 This study presents the first open-source integrated TM framework for

49 reconstructing a metabolic interaction network. This framework can be a powerful tool that helps

50 biologists to extract metabolic events for further reconstruction of a metabolic interaction 51 network. The ME corpus, test corpus, source code, and virtual machine image with pre52 configured software are available at www.sbi.kmutt.ac.th/ preecha/metrecon.

\section{Introduction}

54 The biological literature is vast and quickly growing. Text mining (TM) has become a routine analysis tool for rapidly scanning the entire literature with an essential goal to extract the relationships between named biological entities and concepts. Different examples of TM applications to network construction have been reported, such as protein-protein interactions [1-

4], gene-gene relationships in co-expression and regulatory networks [5-7], and gene-disease relationships $[8,9]$. In addition to a wide range of applications, TM is currently adapted for assisting in compiling relationships of biological data from free texts in biological literature and

61 databases $[10,11]$. In order to face the challenges due to biological complexity, TM tasks have

62 recently advanced from performing simple interaction extraction towards obtaining a better 63 understanding of the semantics behind biological interactions by analyzing associated events.

64 This task is known as event extraction. This development was presented in the form of the 65 BioNLP Shared Task (BioNLP-ST) [12], which is a biological community-wide effort to 66 advance the development of natural language processing (NLP). Recently, BioNLP-ST'13 [13]

67 focused on complex relationships, especially related to the topic of biomolecular reactions, 68 pathways and regulatory networks [14-18]. Focusing on metabolic relationships, the Pathway

69 Curation (PC) Task - BioNLP-ST'13 presented by Ohta et al. [18] introduced an event extraction 70 task setting to account for metabolic pathways. 
71 Despite the great interest in the use of TM tools for the extraction and annotation of biological

72 entities of genes, proteins, or simple chemicals through the curation of events and pathways,

73 there have been limited studies to date at a biological system scale (e.g., events with interaction

74 network).

75 This opens a great challenge for integrating state-of-the-art text mining tasks. Considering 76 closely related prior works [19-23], for example EMPathIE [19], a template-based TM system,

77 was used to extract information about metabolic reactions along with related contextual 78 information (e.g., source organism and pathway name). When evaluated on a corpus, EMPathIE 79 achieved $23 \%$ recall and $43 \%$ precision [19]. Currently, EMPathIE is no longer under active 80 development [19]. In addition, PathBinder [20], which is based on a statistical method derived 81 from syntactic and semantic properties of the biomolecular interactions, was used to locate 82 evidence of interaction between two molecules. PathBinder achieved F-score of $71 \%$ with a 83 predefined dataset. More generic systems may also be used, such as the GeneWays system for 84 extracting, analyzing, visualizing and integrating molecular pathway data [21]. Nonetheless,

85 GeneWays shows lack of any published evaluation of its performance with metabolic pathway 86 data and it is not freely available thus far. Moreover, PathText [22] is a pathway curating 87 environment which integrates pathway visualizers, TM systems, and annotation tools into one 88 unified environment. However, PathText [22] is not openly available. Recently, Czarnecki et al. 89 [23] developed a rule-based approach to reconstruct Escherichia coli metabolic pathways from 90 literature cited in EcoCyc database [24]. Czarnecki et al. achieved recall and precision of 29$9170 \%$ and $14-41 \%$, respectively for metabolic reaction extraction method on evaluated pathways. 92 Their results suggest the possibility of automating the process of extracting metabolic 93 interactions from free texts. 
95 Despite these efforts, many TM tools remain restricted, such as not being freely available (e.g.,

96 GeneWays and Pathtext) or working with merely provided input data (e.g., Pathbinder).

97 Regarding on machine learning (ML) components of TM tools, their primary difference depends

98 on text mining objective and task. These restrictions reduce a tool's ability to integrate a TM

99 framework for automated extracting metabolic interactions from literature. This is a prevalent

100 problem because metabolic studies rely on biological literature. Considering the process of

101 reconstruction of a metabolic interaction network under normal circumstances, biologists depend

102 on the literature and biological databases for annotation and assignment of genes, enzymes,

103 proteins, and metabolites relationships [25-29]. Unless an integrated tool for assisting annotation

104 is available, biologists need to perform gene and functional assignments towards metabolic

105 interaction network using manual curation, which can be both a labor intensive and time

106 consuming task [30, 31].

107

108 With the current state of TM research in a metabolic context, we developed an integrated TM

109 framework to meet the above mentioned challenges. The objective of this study is to perform

110 integration of various TM tools to develop a framework to extract metabolic events and further

111 use the framework for reconstructing a metabolic interaction network. To achieve this task, we

112 initially constructed a Metabolic Entities (ME) corpus composed of a representative set of the

113 metabolic events (i.e., events with a mechanical description of the metabolic interaction). We

114 thereafter took the constructed corpus for further use in the development of a TM framework.

115 The TM framework contains two developed modules. The first module, Metabolic Event

116 Extraction (MEE) module, is used for extracting metabolic events from the constructed corpus. 
117 The second module, Metabolic Interaction Network Reconstruction (MINR) module, is used for

118 reconstructing metabolic interaction networks. For overall evaluation of the integrated TM

119 framework, the predicted entities and pathways were compared to the manually-curated

120 metabolic entities and pathways in the EcoCyc database and the genome-scale metabolic

121 network of Escherichia coli. The integrated TM framework generates metabolic interaction

122 networks in forms of a bipartite metabolic graph and of an enzyme-metabolite interaction pair.

123 The results can be visualized using several types of tools for the task of reconstruction of a

124 metabolic interaction network.

\section{Materials \& Methods}

127 An overview of the proposed integrated TM framework is depicted in Figure 1. It is divided into

128 six main steps: namely (i) construction of ME corpus; (ii) construction of test corpus; (iii)

129 development of MEE module; (iv) development of MINR module; (v) evaluation of integrated

130 TM framework; and (vi) application of integrated TM framework.

132 Construction of Metabolic Entities (ME) corpus

133 The ME corpus is developed and made publicly available at

134 www.sbi.kmutt.ac.th/ preecha/metrecon. The corpus is licensed under a Creative Commons

135 Attribution-ShareAlike 4.0 International License. It is a collection of various abstracts and titles

136 from different databases that have been manually annotated for metabolic events by two

137 annotators who are biologists with different backgrounds. In order to construct the ME corpus,

138 an article list was initially collected from the EcoCyc database (version 16.5). The EcoCyc

139 database was selected as an example source because it contains a comprehensive resource of 
140 biological information for the model organism E.coli K12. It contains manually curated and

141 extensive information (e.g., summary comment, regulatory information, literature citation, and

142 extracted evidence type obtained from thousands of publications) [24]. From this list, articles

143 were randomly selected and their abstracts and titles were then downloaded from the PubMed

144 database for the subsequent annotation process. Considering the process of annotation in each

145 abstract and title, BANNER [32] was first used to annotate gene and protein (GP), as well as

146 metabolite entities according to our annotation guideline

147 (www.sbi.kmutt.ac.th/ preecha/metrecon). Table 1 presents annotated entity types along with

148 reference databases i.e., EcoCyc and ChEBI and Systems Biology Ontology (SBO) ID.

149 Afterwards, manual correction using BRAT [33] by individual domain experts was performed.

150 The metabolic events in the abstracts and titles were annotated according to four types of the

151 metabolic events (i.e., metabolic production, metabolic consumption, metabolic reaction, and

152 positive regulation). Eventually, these annotations were merged to create a final annotation set.

153 For the definition and scope of the metabolic event annotation, the Systems Biology Ontology

154 (SBO) and the Gene Ontology (GO) are considered. Table 2 presents the annotated metabolic

155 event types, arguments and their Ontology ID. A hierarchical representation of the metabolic

156 entities and events is illustrated in Figure 2A. Also, an example of annotation for metabolic

157 entities and events can be seen in Figure 2B. For metabolic entities, the annotation identifies

158 phosphoglucosamine mutase and GlmM as GP entities and glucosamine-1-phosphate and

159 glucosamine-6-phosphate as metabolite entities. For metabolic events, the annotation identifies

160 event words of catalyzes and formation as event types of positive regulation and metabolic

161 reaction, respectively. Note that this ME corpus focuses only on metabolic interactions (i.e.,

162 enzyme-metabolite interactions) throughout metabolic events at the end. Other types of data, 
163 e.g., substrates, products, co-enzymes and co-factors, were considered as metabolites. For

164 discussion of the relation between these entities and event types and the other representations

165 applied in ME corpus, Ohta et al. [18] was used as a reference.

166 To measure an inter-annotation agreement, 20 abstracts and titles were randomly selected as an

167 example case. The two annotators annotated these abstracts and titles according to the annotation

168 guidelines (www.sbi.kmutt.ac.th/ preecha/metrecon). These annotated abstracts and titles were

169 then used to manually construct consensus annotation.

170 The annotated results from the two annotators were compared against the constructed consensus

171 annotation and then the F-score was calculated across different data of GP entities, metabolite

172 entities and events. Additionally, the Cohen's kappa coefficient [34] was also considered as a

173 statistical measure of inter-annotator agreement for GP entities, metabolite entities and events.

174 The overall performance difference between annotators $\mathrm{A}$ and $\mathrm{B}$ is not significant when

175 compared to consensus annotation (F-scores range from 74.07\% to 96.88\%) (see Table 3). Also,

176 the agreement between the two annotators were high across all categories (kappa coefficients

177 range from $0.72-0.96$ ). It is worth noting that multiple interpretations of numerous entities and

178 events between the two annotators may cause high variability of the inter-annotator agreement

179 level.

180 Construction of test corpus

181 The test corpus (www.sbi.kmutt.ac.th/ preecha/metrecon) was constructed from a collection of

182 introduction, abstract and title sections within two Superpathways articles in the EcoCyc and

183 PubMed databases, i.e., the Superpathway of leucine, valine, and isoleucine biosynthesis (18

184 articles) and the Superpathway of pyridoxal 5'-phosphate biosynthesis and salvage (9 articles).

185 The abstract, introduction and title sections of an article were used because they provide a major

186 summary of the research article, supporting statement and theoretical context. It is also noted that 
187 these two selected Superpathways were relatively large compared to others and therefore a

188 considerable number of abstracts, titles and introductions could be collected for test corpus

189 construction. Regarding the annotation process, it was performed as described in the earlier

190 section about the construction of a ME corpus.

191 Development of Metabolic Event Extraction (MEE) module

192 After constructing the ME corpus and test corpus, we developed a MEE module, as illustrated in

193 Figure 3A. This MEE module architecture was divided into two sub-parts, namely text pre-

194 processing and metabolic event extraction as described below.

195 Text pre-processing

196 The input text (e.g., abstract) as seen in Figure 3A-a1 was split into sentences using the GENIA

197 Sentence Splitter [35]. Each sentence was then parsed using the McClosky-Charniak-Johnson re-

198 ranking parser [36]. All sentences with more than 100 tokens were skipped and the parser

199 produced a n-best list of the $n$ most likely parses of the sentence $(n=50$ was set as the default

200 parameters in this study). Afterwards, the sentence was converted into collapsed dependency

201 parse using the Stanford parser [37]. Next, BANNER [32] was used for detecting all possible GP

202 entities in the sentence. To carry out the subsequent MINR module development work on the

203 metabolic interaction network, we selected only enzyme entities out of all possible GP entities

204 throughout metabolic events by using event word, EcoCyc enzyme name and manual curation.

205 For metabolite entities detection, BANNER trained on the yeast metabolite corpus [38] was

206 chosen based on our previous performance evaluation [39].

207 Metabolic event extraction

208 We retrofitted the Turku Event Extraction System (TEES) by reconfiguration of the entities and

209 the event types as well as retraining TEES in order to support metabolites and metabolic event 
210 extraction. Notably, we selected TEES because of its overall performance and ability to handle

211 large-scale datasets [16, 40]. TEES is an event classification tool, which utilizes various features

212 from syntax analysis, such as tokens and dependency graphs to identify the probability of

213 metabolic interaction between two entities [41]. The TEES parameter was estimated from its

214 internal Support Vector Machine while training using a grid search. The retrofitted TEES can be

215 found in the virtual machine image available at www.sbi.kmutt.ac.th/ preecha/metrecon.

216 Development of Metabolic Interaction Network Reconstruction (MINR) module

217 Once MEE module was developed, we further developed a MINR module. We divided MINR

218 module into two sub-sections: the mapping from metabolic event to metabolic interaction and

219 reconstructing the metabolic interaction network by combinations of individual metabolic

220 interactions.

221 Mapping from metabolic event to metabolic interaction

222 Extracted metabolic events together with enzyme and metabolite entities obtained from the MEE

223 module (Figure 3A-a2 and Figure 3A-a3) were initially converted into a graph called an event

224 interaction graph in the MINR module (Figure 3B-b1). This step represented the whole event in a

225 graph format. The event interaction graph was then transformed into a metabolic interaction

226 graph (Figure 3B-b2). To simplify the events for interaction extraction, the redundancy of the

227 extracted events across articles was eliminated by rearrangement and deletion of nodes based on

228 a predefined rule. Additional file 1 shows the pseudocode used for the MINR module

229 development in converting an event interaction graph to a metabolic interaction graph, as

230 illustrated in Figure 3B-b1 to Figure 3B-b2. The extracted enzyme-metabolite interaction pair

231 could then be eventually obtained, as shown in Figure 3B-b3.

232 


\section{Reconstructing metabolic interaction network}

234 After mapping from the metabolic event to metabolic interaction, all possible extracted enzyme-

235 metabolite interaction pairs were pooled together. Subsequently, all unique enzyme-metabolite

236 interaction pairs were merged together and then mapped onto a metabolic interaction network.

237 Metabolites were connected through shared enzymes. The resulting metabolic interaction

238 network was enforced to be a bipartite metabolic graph (i.e., enzymes and metabolites). In the

239 case of an incomplete metabolic interaction (e.g., no enzyme for the connecting metabolites), a

240 missing node was filled with a proxy node in order to conform to a bipartite metabolic structure.

\section{Evaluation of integrated TM framework}

242 To evaluate the integrated TM framework, we assessed the MEE module and the MINR module,

243 separately as described below. MEE module evaluation on three critical sub-parts: ME corpus,

244 ME corpus size and test corpus was performed. In each sub-part, we calculated performance

245 based on standard precision, recall, and F-score as performance measures [42].

246 To compare the obtained results with manually curated entities, we applied the sloppy span

247 matching criterion to entities, which means that entities must match the types, but are not

248 required to exactly match the entities boundaries [23]. For metabolic events, the comparison

249 criterion that is termed approximated boundary matching [43] was used. In particular, the three

250 criteria used were: (i) identical metabolic event type, (ii) sloppy span matching between

251 metabolic event trigger span, and (iii) at least one matching or all matching of arguments. We

252 used these criteria in this study due to strong supporting evidence that they are better in terms of

253 information retrieval than the alternative exact matching criterion, where boundaries between

254 two entities are required to match exactly $[2,44]$. 
255 MEE module evaluation on ME corpus

256 We performed five-fold cross-validation on the abstracts and titles for error estimation of MEE

257 module. The total number $(D)$ of the abstracts and the titles within ME corpus was randomly

258 partitioned into five approximately equal numbers $\left(D_{1}, D_{2}, \ldots, D_{5}\right)$. An individual fold was

259 iteratively left out and used as the testing dataset while the remaining data were used as the

260 training dataset.

261 MEE module evaluation on ME corpus size

262 In addition to assessing the MEE module using the whole ME corpus constructed in this work,

263 we also evaluated the effect of various corpus sizes. For this part, we performed five-fold cross-

264 validation on subsets of the ME corpus with different abstract sizes. To create these subsets, 100,

265150 , and 200 abstracts and titles were randomly selected from the constructed ME corpus. To

266 this end, we compared the results with that obtained from the whole corpus of 271 abstracts and

267 titles, which was used to determine an upper bound of F-score.

MEE module evaluation on test corpus

269 We evaluated the entities prediction performance of the MEE module using the test corpus as a

270 reference. That is, we ran entities prediction using the MEE module on the test corpus. The

271 predicted results of GP and metabolite entities were then compared to the manually-curated

272 entities in test corpus.

273 MINR module evaluation on test corpus

274 A list of manually-curated metabolic interactions was initially prepared for the MINR module

275 evaluation on the test corpus as provided in Additional file 2. After applying the MINR module

276 on the test corpus for further metabolic interaction network reconstruction, the predicted results

277 were then compared to the manually-curated metabolic interactions list (Additional file 2) for

278 evaluation throughout calculation of the recall, the precision, and the F-score. 
279 Application of integrated TM framework

280 To demonstrate the application of the integrated TM framework, we presented two case studies.

281 The first case study showed a comparative analysis of our integrated TM framework with

282 another TM system developed by Czarnecki et al. [23] on the test corpus for reconstruction of

283 the Superpathway of leucine, valine, and isoleucine biosynthesis. To elaborate, we ran our

284 integrated TM framework on the test corpus to extract the metabolic events and mapped them to

285 metabolic interactions for the Superpathway reconstruction. Once completed, we then performed

286 a comparative analysis of the reconstructed network with the other results achieved by the TM

287 system developed by Czarnecki et al. [23].

288 For the second case study, the integrated TM framework with EcoCyc extraction was applied to

289 reconstruct a metabolic interaction network. EcoCyc extraction is a collection list of references

290 from the EcoCyc database (2,373 abstracts and titles). In brief, we first ran the integrated TM

291 framework on EcoCyc extraction in order to extract a list of enzyme, metabolite, and enzyme-

292 metabolite interactions association with metabolic events. Also, we ran the integrated TM

293 framework on the ME corpus for performance comparison. To evaluate the performance, the

294 predicted entities in terms of enzymes, metabolites, and enzyme-metabolite interactions were

295 compared to the manually-curated metabolic entities in the EcoCyc database for calculating the

296 precision. A published genome-scale metabolic network of E. coli K-12 MG1665 (iJO1366) [45]

297 was also used as the manually-curated metabolic pathways for metabolic interaction network

298 reconstruction.

299 The integrated TM framework could be run using the virtual machine image on one of Mac,

300 Windows, or Linux system with the pre-configured software available at

301 www.sbi.kmutt.ac.th/ preecha/metrecon. The source code in the virtual machine is licensed

302 under Apache License 2.0. 


\section{Results and Discussion}

304 The ME corpus and an integrated TM framework for the reconstruction of a metabolic

305 interaction network were developed in this study. The results and discussion are provided below.

306 ME corpus statistics

307 Our constructed ME corpus consists of annotated GP, metabolite entities, and metabolic events.

308 Table 4 shows the basic statistics for the constructed ME corpus. Of the 271 abstracts and titles

309 selected from the PubMed database (see Methods), we found a total number of 2,513 entities for

310 GP and 1,898 entities for metabolite, corresponding to 9.27 and 7.00 entities per abstract for GP

311 and metabolite, respectively.

312 In addition, we further examined the basic statistics on the average number of arguments per

313 metabolic event. As also shown in Table 4, the metabolic reaction event has an average number

314 of 2.12 arguments per metabolic event. This is higher than the average number of arguments in

315 metabolic production event (1.10) and metabolic consumption event (1.22). In order to further

316 express the word preference in the metabolic event, we inspected common words in the ME

317 corpus. The top ten list of event words identified in the ME corpus is presented in Additional file

318 3. Interestingly, we found that a major portion of these common words $(45.84 \%$ of total

319 metabolic events) are involved in the generic description of biological processes (e.g., Catalyzes,

320 Biosynthesis, Synthesis, Formation, Conversion, Utilization, Catalyzed, Catalyze, and

321 Metabolism). These results showed that most of the event words in the metabolic event were

322 centred around a small set of general keywords. However, it is possible to deduce the types of

323 enzymatic reactions using name of substrate and product in some cases, e.g. the formation of

324 glucosamine-1-phosphate (product name) from glucosamine-6-phosphate (substrate name)

325 (Figure 2B) suggests a phosphorylation reaction. 
326 Performance of MEE module on ME corpus

327 Using five-fold cross-validation on the ME corpus, the recall, the precision, and the F-score were

328 calculated for each metabolic event type as measures for the overall performance evaluation.

329 Table 5 shows these measures for each metabolic event type as well as for the total event type

330 which indicates the sum of all metabolic event types. That is, the measures of total event type

331 were calculated by summation of the individual true positives, false positives, and false negatives

332 for each metabolic event type.

333 The F-scores of the events of metabolic production (59.15\%) and metabolic consumption

$334(48.59 \%)$ turned out to be higher than both of the events of metabolic reaction $(28.32 \%)$ and

335 positive regulation $(36.69 \%)$. It is intuitive to think that complex events (i.e., two or more

336 arguments) as found in metabolic reaction and positive regulation are harder to be classified than

337 simple events (i.e., one argument) as found in metabolic production and metabolic consumption.

338 In such a case, when the average number of arguments per metabolic event is high, a low F-score

339 is clearly shown (see Tables 4 and 6) as in the example of the metabolic reaction and the positive

340 regulation. These above-mentioned results are strongly supported by earlier works in Pathway

341 Curation (PC) task - BioNLP-ST'13 and BioNLP-ST'11 [18, 46]. In particular, the F-scores

342 achieved from positive regulation event

343 between PC task - BioNLP-ST'13 [18] and our study were compared.

344 Consequently, the F-scores were similar with values of 39.23 and 36.69 ,

345 respectively.

346 Performance of MEE module on different ME corpus sizes

347 To assess the effect of ME corpus sizes, different subsets of 100, 150, and 200 abstracts and titles

348 were randomly extracted and compared to the whole ME corpus size of 271 abstracts and titles.

349 Each subset was evaluated three times, and the results shown were averages (Figure 4). The 
350 corresponding recall, precision and F-score were compared in the form of learning curves in

351 Figure 4 using five-fold cross-validation. Expectedly, the performance of MEE module with the

352 largest corpus size was better than that of the smaller corpus sizes in all possible cases. Clearly,

353 the best recall and F-score were obtained with the whole corpus size of 271 abstracts and titles

354 (Figures $4(\mathrm{~A})$ and $(\mathrm{C})$ ). In general, the trend was improved performance with a larger corpus

355 size for these two measures. A similar trend was observed for the precision measure, except for

356 the case of metabolic production which showed no dependence on corpus size (Figure 4 (B)).

357 From the overall results, we suggest that a minimum of 150 abstracts and titles should be used

358 for development of a ME corpus. Note that the regularity of the metabolic event description is

359 applied for development of ME corpus for easier event extraction.

\section{Performance of MEE module on test corpus}

361 As a further assessment of the proposed integrated TM framework, a constructed test corpus was

362 used for performance evaluation of the MEE module. Basic statistics of test corpus can be seen

363 in Table 4. It contained 27 articles related to two Superpathways from the EcoCyc database (see

364 Methods). At first, we evaluated MEE module on the test corpus using GP and metabolite entity

365 tagging. As shown in Table 6, the precision, recall, and F-score of the entity tagger for GP and

366 metabolite were very high for more than $80 \%$ of GP and metabolite entities identified for the

367 Superpathway of leucine, valine, and isoleucine biosynthesis. However, the recall and F-score of

368 the entity tagger for metabolite was lower than our expectations for the Superpathway of

369 pyridoxal 5'-phosphate biosynthesis and salvage. The recall showed less than $70 \%$, and the

370 F-score showed less than $80 \%$. These results seem to indicate that the entity tagger (i.e

371 BANNER) has a weakness in detecting metabolite entities in an abbreviated form (e.g.,

372 Pyridoxine (PN), Pyridoxal (PL), and 4-hydroxy-l-threonine phosphate (HTP)). 
373 Performance of the MINR module on test corpus

374 We evaluated the performance of the MINR module using the test corpus. In terms of enzyme-

375 metabolite interaction, we found that the MINR module showed high performance as presented

376 in Table 7. The reconstructed results from the MINR module were then compared with manually

377 curated metabolic interactions list (see Additional file 2). As shown in Table 7, for the two

378 Superpathways, the precisions were $80-90 \%$, the F-scores were $70-80 \%$, and the recalls were 60 -

$37970 \%$. These results suggest that the MINR module performed well for the mapping of enzyme-

380 metabolite interactions and can be further used for reconstruction of metabolic interaction

381 networks. Nonetheless, there were still missing interactions which could not be identified by the

382 MINR module. These could be because MINR module was unable to capture the metabolic

383 events that were implicit in the text as seen in the example of PMID-13405870 (see Additional

384 file 4). Moreover, it was also unable to extract exact precedence relationships among metabolic

385 events as seen in the example of PMID-13727223 (see Additional file 4). Further improvements

386 are planned for MINR module after taking into account these limitations.

387 The integrated TM framework for reconstructed metabolic interaction network

388 As mentioned in the Methods section, two case studies were used to evaluate the integrated TM

389 framework. For the first case study, as shown in Figure 5, our integrated TM framework on the

390 test corpus successfully extracted 11 entities of enzymes and metabolites as well as 10 enzyme-

391 metabolite interactions for reconstruction of the Superpathway of leucine, valine, and isoleucine

392 biosynthesis.

393 To elaborate how a biologist can apply our integrated TM framework for reconstruction of the

394 Superpathway of leucine, valine, and isoleucine biosynthesis, we show two example sentences

395 extracted from PMID-1646790 that were obtained from MEE and MINR modules. The examples

396 are described below. 
397 Example 1: "leucine synthesis by the tyrosine-repressible transaminase in Escherichia coli $\mathrm{K}$ 398 12”

399 Example 2: "2-KIC amination by the tyrB-encoded transaminase and also by the aspC-and 400 avtA-encoded transaminases"

401 For explanation of example 1, the MEE module identifies enzyme (tyrosine-repressible 402 transaminase) and metabolite (leucine) throughout metabolic production event. Considering 403 example 2, the MEE module identifies enzyme (tyrB-encoded transaminase) and metabolite (2404 KIC) throughout metabolic consumption event. To the end, the MINR module obtains enzyme405 metabolite interactions by combining the metabolic production and consumption events from 406 examples 1 and 2, respectively. As a result, 2-KIC can be converted to leucine by tyrB-encoded 407 transaminase (tyrosine-repressible transaminase). Full details of enzymes, metabolites, and 408 enzyme-metabolite interactions can be seen in Additional file 5.

409 Comparing these results to those obtained by a TM system developed by Czarnecki et al. [23],

410 we found that a similar number of correctly extracted entities of enzymes and metabolites and 411 enzyme-metabolite interactions were obtained (Figure 5). As can be seen, the results achieved

412 from both systems are able to extract a different part of network suggesting that the combination

413 of the results and biological intepretation would be an interesting option for a biologist who

414 searches for an alternative way for reconstructing a network.

415 For the second case study involving large-scale data extraction from EcoCyc, the results are 416 shown in Table 8. We found the precisions for this EcoCyc extraction data in terms of enzymes 417 (69.93\%), metabolites (70.63\%), and enzyme-metabolite interactions (46.71\%). After comparing 418 these precisions to the similar results gained from constructed ME corpus (271 abstracts and 419 titles), we found that the EcoCyc extraction data showed a higher number of false positives. 420 Based on our manual inspection, one source of false positives came from mentions of enzymes, 
421 metabolites, or enzyme-metabolite interactions in other species that were not from $E$. coli despite

422 the fact that our framework was trained using E. coli abstracts and titles. However, this is

423 favorable for the real-world usage since it shows the generality of our method can capture all

424 generic mentioned reactions in text. Another note is that we did not deploy a normalization

425 method in our evaluation, and this might not correctly reflect the performance of the real-world

426 large-scale extraction where the normalization method is critical. Nevertheless, these results

427 illustrate that our constructed ME corpus within the integrated TM framework is solid and can be

428 used as a representative dataset for large-scale data extraction with applications for building

429 metabolic interaction databases and networks as well as for knowledge discovery tasks. The

430 proposed integrated TM framework application is summarized in Figure 6.

\section{Conclusions}

432 This study reports the first open-source integrated TM framework for

433 reconstructing a metabolic interaction network. Here, we constructed a ME corpus, a MEE

434 module and a MINR module within an integrated TM framework. Expectedly, the ME corpus

435 has been successfully used for simplified detection of GP, metabolites entities throughout

436 metabolic events. In addition, we have shown that our proposed framework successfully

437 extracted a metabolic interaction, and it can be used as a scaffold for futher reconstruction of a

438 large-scale metabolic interaction network.

439 From the overall performance evaluation, the two developed modules within the framework

440 performed well. Using five-fold cross-validation in the MEE module on the ME corpus, F-scores

441 of $59.15 \%$ and $48.59 \%$ were obtained for metabolic production and consumption, respectively.

442 This indicates practical performance of the MEE module in the detection of metabolic entities. In

443 the comparative study of corpus sizes, the MEE module showed a high F-score and a high recall 
444 when the size increased. The correlation of its overall performance implied the extensibility of

445 the integrated TM framework which could be achieved by increasing the size of the corpus.

446 With regards to the entity tagger for GP and metabolites on the test corpus, the overall

447 performance showed F-score for more than $80 \%$ as illustrated for the Superpathway of leucine,

448 valine, and isoleucine biosynthesis. Concerning on the evaluated MINR module on the test

449 corpus, F-score of above $70 \%$ was achieved in mapping of enzyme-metabolite interactions

450 through network reconstruction. Furthermore, the integrated TM framework could be used to

451 successfully reconstruct the Superpathway of leucine, valine, and isoleucine biosynthesis with

452 comparable performance to another TM system in terms of enzymes, metabolites, and enzyme-

453 metabolite interactions. Finally, when the integrated TM framework was applied with an EcoCyc

454 extraction for reconstructing a metabolic interaction network, reasonable precisions of enzyme

455 (69.93\%), metabolite (70.63\%) and enzyme-metabolite interaction (46.71\%) were obtained. We

456 believe that this study can be the first such TM framework for developing further automation

457 tools for assisting in metabolic network reconstruction. In addition, this TM framework is

458 beneficial for general usability because it can run on Linux, Window and MAC systems. Based

459 on our investigations, finer details of metabolic interaction, such as types of interactions,

460 locations, pathways and species could be deduced from metabolic events and linguistic patterns.

461 This rich information would allow us to build a more accurate representation of metabolic

462 interactions and more sophisticated metabolic network reconstruction. For a future plan, other

463 databases, e.g., MetaCyc, contain thousands of different organisms which can be used for

464 evaluation and application of an integrated TM framework for a species-specific metabolic

465 interaction network. Full text of paper is recommended for future development of corpus. Due to

466 the limitation of the current tool (e.g. BANNER) used in this study, the NLP-based approach 
467 should be further investigated and implemented for increasing the overall performance of the

468 integrated TM framework. An API for implementation of user-defined algorithms will also be

469 provided, including a user interface and a web-service for event annotation.

470 List of abbreviations used

471 TM: Text Mining; PN: Pyridoxine; PL: Pyridoxal; 4-hydroxy-1-threonine phosphate: HTP;

472 TEES: Turku Event Extraction System; GP: Gene and Protein; SD: Standard deviation; GREC:

473 Gene Regulation Event Corpus; BioNLP-ST: BioNLP Shared Task; NLP: Natural Language

474 Processing; ME: Metabolic Entities; MEE module: Metabolic Event Extraction module; MINR

475 module: Metabolic Interaction Network Reconstruction module.

476 Acknowledgements

477 We would like to thank Mr. Yutthanattee Tohreh for assisting in the manual annotation process

478 and Mr. Sean Kortschot for proofreading the draft version of the manuscript. We also would like 479 to thank Mr. David H. Cook for proofreading the revised version of manuscript.

\section{References}

481 1. Saetre R, Yoshida K, Miwa M, Matsuzaki T, Kano Y, Tsujii J: Extracting protein 482 interactions from text with the unified AkaneRE event extraction system. IEEE/ACM 483 Transactions on Computational Biology and Bioinformatics 2010, 7:442-53.

484 2. Kabiljo $\mathrm{R}$, Clegg $\mathrm{AB}$, Shepherd $\mathrm{AJ}$ : A realistic assessment of methods for extracting 485 gene/protein interactions from free text. BMC Bioinformatics 2009, 10:233. 
486 3. Airola A, Pyysalo S, Björne J, Pahikkala T, Ginter F, Salakoski T: All-paths graph kernel

487 for protein-protein interaction extraction with evaluation of cross-corpus learning. $B M C$ 488 Bioinformatics 2008, 9 Suppl 11(Suppl 11):S2.

489 4. Srihari S, Leong HW: A survey of computational methods for protein complex prediction 490 from protein interaction networks. Journal of Bioinformatics and Computational Biology $491 \quad 2012: 1230002$.

5. Rodríguez-Penagos C, Salgado H, Martínez-Flores I, Collado-Vides J: Automatic 493 reconstruction of a bacterial regulatory network using Natural Language Processing. $B M C$ 494 Bioinformatics 2007, 8:293. networks. Interdisciplinary Sciences: Computational Life Sciences 2009, 1:179-86. Salakoski T, Van de Peer Y, Ginter F: Large-scale event extraction from literature with multi-level gene normalization. PLoS One 2013, 8:e55814.

500 8. Bell L, Chowdhary R, Liu JS, Niu X, Zhang J: Integrated bio-entity network: a system for 501 biological knowledge discovery. PLoS One 2011, 6:e21474.

502 9. Ozgür A, Vu T, Erkan G, Radev DR: Identifying gene-disease associations using centrality on a literature mined gene-interaction network. Bioinformatics 2008, 24:i277-85. 
504 10. Hirschman L, Burns GAPC, Krallinger M, Arighi C, Cohen KB, Valencia A, Wu CH, Chatr-

505 Aryamontri A, Dowell KG, Huala E, Lourenco A, Nash R, Veuthey A-L, Wiegers T, Winter

506 AG: Text mining for the biocuration workflow. Database 2012, 2012:bas020.

507 11. Neves M, Damaschun A, Mah N, Lekschas F, Seltmann S, Stachelscheid H, Fontaine J-F,

508 Kurtz A, Leser U: Preliminary evaluation of the CellFinder literature curation pipeline for

509 gene expression in kidney cells and anatomical parts. Database 2013, 2013:bat020.

510 12. Kim JD, Wang Y, Takagi T, Yonezawa A: Overview of Genia Event Task in BioNLP

511 Shared Task 2011. Proceedings of BioNLP Shared Task 2011 Workshop; 2011:7-15.

512 13. Kim J-D, Wang Y, Yasunori Y: The Genia Event Extraction Shared Task, 2013 Edition -

513 Overview. In Proceedings of BioNLP Shared Task 2013 Workshop. Sofia, Bulgaria: Association

514 for Computational Linguistics; 2013.

515 14. Landeghem S Van, Ginter F: EVEX: a PubMed-scale resource for homology-based 516 generalization of text mining predictions. Proceedings of BioNLP 2011 Workshop; 2011.

517 15. McClosky D, Riedel S, Surdeanu M, McCallum A, Manning CD: Combining joint models

518 for biomedical event extraction. BMC Bioinformatics 2012, 13 Suppl 1(Suppl 11):S9.

519 16. Gerner M, Sarafraz F, Bergman CM, Nenadic G: BioContext: an integrated text mining

520 system for large-scale extraction and contextualization of biomolecular events. 521 Bioinformatics 2012, 28:2154-61.

522 17. Bossy R, Bessières P, Nédellec C: BioNLP Shared Task 2013 - An overview of the Genic

523 Regulation Network Task. In Proceedings of BioNLP Shared Task 2013 Workshop; 2013. 
524 18. Ohta T, Pyysalo S, Rak R, Rowley A, Chun H-W, Jung S-J, Choi S-P, Ananiadou S:

525 Overview of the Pathway Curation (PC) task of BioNLP Shared Task 2013. In Proceedings

526 of BioNLP Shared Task 2013 Workshop; 2013.

527 19. Humphreys K, Demetriou G, Gaizauskas R: Two applications of information extraction to

528 biological science journal articles: enzyme interactions and protein structures. Pacific

529 Symposium on Biocomputing 2000, 513:505-516.

530 20. Zhang L, Berleant D, Ding J, Cao T, Syrkin Wurtele E: PathBinder--text empirics and

531 automatic extraction of biomolecular interactions. BMC Bioinformatics 2009, 10 Suppl

532 1(Suppl 11):S18.

533 21. Rzhetsky A, Iossifov I, Koike T, Krauthammer M, Kra P, Morris M, Yu H, Duboué PA,

534 Weng W, Wilbur WJ, Hatzivassiloglou V, Friedman C: GeneWays: a system for extracting,

535 analyzing, visualizing, and integrating molecular pathway data. Journal of biomedical

536 informatics 2004, 37:43-53.

537 22. Kemper B, Matsuzaki T, Matsuoka Y, Tsuruoka Y, Kitano H, Ananiadou S, Tsujii J:

538 PathText: a text mining integrator for biological pathway visualizations. Bioinformatics

$5392010,26: i 374-81$.

540 23. Czarnecki J, Nobeli I, Smith AM, Shepherd AJ: A Text-Mining System for Extracting

541 Metabolic Reactions from Full-Text Articles. BMC Bioinformatics 2012, 13:172.

542 24. Keseler IM, Mackie A, Peralta-Gil M, Santos-Zavaleta A, Gama-Castro S, Bonavides-

543 Martínez C, Fulcher C, Huerta AM, Kothari A, Krummenacker M, Latendresse M, Muñiz- 
544 Rascado L, Ong Q, Paley S, Schröder I, Shearer AG, Subhraveti P, Travers M, Weerasinghe D,

545 Weiss V, Collado-Vides J, Gunsalus RP, Paulsen I, Karp PD: EcoCyc: fusing model organism

546 databases with systems biology. Nucleic acids research 2013, 41:D605-12.

547 25. Bordbar A, Palsson BØ: Using the reconstructed genome-scale human metabolic 548 network to study physiology and pathology. Journal of internal medicine 2012, 271:131-41.

549 26. Feist AM, Herrgård MJ, Thiele I, Reed JL, Palsson BØ: Reconstruction of biochemical 550 networks in microorganisms. Nature reviews Microbiology. 2009, 7:129-43.

551 27. Heavner BD, Smallbone K, Barker B, Mendes P, Walker LP: Yeast 5 - an expanded 552 reconstruction of the Saccharomyces cerevisiae metabolic network. BMC Systems Biology $5532012,6: 55$.

554 28. Poolman MG, Miguet L, Sweetlove LJ, Fell DA: A genome-scale metabolic model of 555 Arabidopsis and some of its properties. Plant physiology 2009, 151:1570-81.

556 29. Liu J, Gao Q, Xu N, Liu L: Genome-scale reconstruction and in silico analysis of 557 Aspergillus terreus metabolism. Molecular BioSystems 2013, 9:1939-48.

558 30. Andersen MR, Nielsen ML, Nielsen J: Metabolic model integration of the bibliome, 559 genome, metabolome and reactome of Aspergillus niger. Molecular Systems Biology 2008, $560 \quad 4: 178$

561 31. Baumgartner WA, Cohen KB, Fox LM, Acquaah-Mensah G, Hunter L: Manual curation is 562 not sufficient for annotation of genomic databases. Bioinformatics 2007, 23:i41-8. 
563 32. Leaman R, Gonzalez G: BANNER: an executable survey of advances in biomedical

564 named entity recognition. Pacific Symposium on Biocomputing Pacific Symposium on

565 Biocomputing 2008:652-63.

566 33. Stenetorp P, Pyysalo S, Topic G: BRAT: a Web-based Tool for NLP-Assisted Text

567 Annotation. In Proceedings of the 5th International Symposium on Semantic Mining in 568 Biomedicine; 2012.

569 34. Cohen J: A Coefficient of Agreement for Nominal Scales. Educational and Psychological 570 Measurement 1960, 20:37-46.

571 35. Sætre R, Yoshida K, Yakushiji A: AKANE system: protein-protein interaction pairs in 572 BioCreAtIvE2 challenge, PPI-IPS subtask. In Proceedings of the Second BioCreative 573 Challenge Workshop; 2007.

574 36. McClosky D, Charniak E, Johnson M: Effective self-training for parsing. In Proceedings of 575 the main conference on Human Language Technology Conference of the North American 576 Chapter of the Association of Computational Linguistics; 2006:152-159.

577 37. Marneffe M, Maccartney B, Manning C: Generating Typed Dependency Parses from 578 Phrase Structure Parses. In Proceedings of LREC-06; 2006:449 - 454.

579 38. Nobata C, Dobson PD, Iqbal SA, Mendes P, Tsujii J, Kell DB, Ananiadou S: Mining 580 metabolites: extracting the yeast metabolome from the literature. Metabolomics 2011, 7:94581101. 
582 39. Patumcharoenpol P, Chan J, Meechai A, Shen B, Vongsangnak W: Bibliome Mining

583 Platform and Application for Building Metabolic Interaction Network. Procedia Computer

584 Science 2012, 11:55-62.

585 40. Björne J, Landeghem S Van, Pyysalo S: PubMed-Scale Event Extraction for Post-

586 Translational Modifications, Epigenetics and Protein Structural Relations. In Proceeding

587 BioNLP '12 Proceedings of the 2012 Workshop on Biomedical Natural Language Processing;

588 2012: 82-90.

589 41. Björne J, Salakoski T: Generalizing Biomedical Event Extraction. In Proceedings of the 590 BioNLP 2011 Workshop; 2011: 183-191.

591 42. Rijsbergen C Van: Information Retrieval. 2nd edition. Butterworth, London; 1979.

592

593

594 Task; 2009:1-9.

595

596

597

598

599

600

601 2011, 7:535.
44. Shepherd A, Kabiljo R: Protein name tagging in the immunological domain. In Proceedings of the Third Symposium on Semantic Mining in Biomedicine; 2008.

45. Orth JD, Conrad TM, Na J, Lerman JA, Nam H, Feist AM, Palsson BØ: A comprehensive genome-scale reconstruction of Escherichia coli metabolism. Molecular systems biology

46. Kim J-D, Pyysalo S, Ohta T, Bossy R, Nguyen N, Tsujii J: Overview of BioNLP Shared Task 2011. In Proceedings of BioNLP Shared Task 2011 Workshop; 2011:1-6. 


\section{Figures}

603 Figure 1 - A schematic diagram outlining the development steps for the proposed 604 integrated TM framework.

605 Note: Metabolic entities corpus size means the effect of different corpus sizes on performance of

606 MEE module within the integrated TM framework.

607 Figure 2 - A schematic annotation of metabolic entities and events

608 (A) Hierarchical representation of metabolic entities and events. (B) An example of metabolic

609 entities and events annotation.

610 Figure 3 - An illustration showing the process implementation of the proposed integrated 611 TM framework.

612 (A) Metabolic Event Extraction (MEE) module; (a1) Input text for MEE module, (a2) Output 613 text from MEE module, (a3) Text output visualization using BRAT [33]. (B) Metabolic 614 Interaction Network Reconstruction (MINR) module; (b1) Intermediate graphical output from 615 MINR module, (b2) Graphical output from MINR module, (b3) enzyme-metabolite interactions 616 pair from MINR module.

617 Figure 4 - Learning curves showing performance of the Metabolic Event Extraction 618 (MEE) module on the different corpus sizes using five-fold cross-validation.

619 Each of metabolic event types and total are plotted against (A) Recall, (B) Precision, and (C) F620 score. The total was calculated by summation of the individual true positives, false positives, and 621 false negatives for each of metabolic event types.

622 Figure 5 - Comparative analysis of two TM systems on test corpus for the Superpathway 623 reconstruction of leucine, valine, and isoleucine biosynthesis.

624 (A) Our integrated TM framework (MEE and MINR modules), (B) TM system developed by 625 Czarnecki et al. [23]. The extracted interactions were overlaid to the reference metabolic 626 pathway from the EcoCyc database. A grey node means correct extraction. A white node means 627 incorrect extraction. An edge with an arrow means correct extraction. An edge with a dash arrow 628 means incorrect extraction. This bipartite metabolic graph is created using Cytoscape version 629 3.0.1. * The extracted enzyme-metabolite interaction indicates the number of binary interaction. $630{ }^{\#}$ The number in parenthesis is a percentage of extracted mentions/events in comparison to the 631 reference metabolic pathway from the EcoCyc database.

632 Figure 6 - The integrated TM framework application.

633 
634

\section{Additional files}

636 Additional file 1 - Pseudocode for conversion from event interaction graph to metabolic 637 interaction graph (Figure 3B-b1 and 3B-b2) used in MINR module development.

638 Additional file 2 - List of manually metabolic interaction used for MINR module evaluation on 639 test corpus.

640 Additional file 3 - Top ten list of event words identified in the ME corpus

641 Additional file 4 - Example results of metabolic interaction list achieved between manual 642 curation and MINR module.

643 Additional file 5 - The reconstructed Superpathway of leucine, valine, and isoleucine 644 biosynthesis from MEE and MINR modules.

645

646

647 


\section{Table 1 (on next page)}

Table 1 - Description of entity types 
1 Table 1 - Description of entity types

\begin{tabular}{lll}
\hline Entity types & Reference & Ontology Id \\
\hline Gene or Protein (GP) & Ecocyc & SBO:0000246 \\
Metabolite & ChEBI & SBO:0000247 \\
\hline
\end{tabular}

2 
Table 2 (on next page)

Table 2 - Description of metabolic event types 
1 Table 2 - Description of metabolic event types

\begin{tabular}{|c|c|c|c|}
\hline Event type & Argument & Description & Ontology ID \\
\hline Metabolic production & $\begin{array}{l}\text { Theme: Metabolite, } \\
\text { Cause: Enzyme }\end{array}$ & $\begin{array}{l}\text { Metabolic event that } \\
\text { results in formation of } \\
\text { metabolite. }\end{array}$ & SBO:0000176 \\
\hline Metabolic consumption & $\begin{array}{l}\text { Theme: Metabolite, } \\
\text { Cause: Enzyme }\end{array}$ & $\begin{array}{l}\text { Metabolic event that } \\
\text { results in consumption } \\
\text { of metabolite. }\end{array}$ & SBO:0000176 \\
\hline Metabolic reaction & $\begin{array}{l}\text { Theme: Metabolite, } \\
\text { Cause: Enzyme }\end{array}$ & $\begin{array}{l}\text { Metabolic event that } \\
\text { results in conversion of } \\
\text { metabolite. }\end{array}$ & SBO:0000176 \\
\hline Positive regulation & $\begin{array}{l}\text { Theme: Event, } \\
\text { Cause: Enzyme }\end{array}$ & $\begin{array}{l}\text { Enzyme related to a } \\
\text { process that positively } \\
\text { regulates a metabolic } \\
\text { event. }\end{array}$ & $\begin{array}{l}\text { GO:0048518, } \\
\text { GO:0044093 }\end{array}$ \\
\hline
\end{tabular}

2 
Table 3(on next page)

Table 3 - Inter-annotator agreement of constructed ME corpus 
1 Table 3 - Inter-annotator agreement of constructed ME corpus

\begin{tabular}{lccc}
\hline Data & Annotator A & Annotator B & $\begin{array}{c}\text { Annotator A/ } \\
\text { Annotator B }\end{array}$ \\
\hline Entities & F-score (\%) & F-score (\%) & $\begin{array}{c}\text { Cohen's kappa } \\
\text { coefficient }\end{array}$ \\
\hline GP & 96.17 & 96.03 & 0.96 \\
Metabolite & 93.58 & 91.72 & 0.92 \\
\hline Events & & & 0.72 \\
\hline Metabolic production & 90.24 & 83.33 & 0.74 \\
Metabolic consumption & 96.88 & 85.71 & 0.90 \\
Metabolic reaction & 74.07 & 75.47 & 0.77 \\
Positive regulation & 85.71 & 94.44 & \\
\hline
\end{tabular}




\section{Table 4(on next page)}

Table 4 - Basic statistics of ME corpus and test corpus 
2 Table 4 - Basic statistics of ME corpus and test corpus

\begin{tabular}{ll}
\hline Metabolic entities corpus & \\
\hline Features & \# Units \\
\hline Abstract & 271 \\
Sentence & 2,288
\end{tabular}

Entities type

Metabolite

GP

Total

Events type

Metabolic production

Metabolic consumption

Metabolic reaction

Positive regulation

Total
Metabolic entities count

$1,898\left(7.00^{*}, 6.52\right)$

$2,513\left(9.27^{*}, 8.02\right)$

$4,411\left(16.28^{*}, 10.74\right)$

\begin{tabular}{ll}
\hline Test corpus & \# Units \\
\hline Features & 27 \\
\hline Abstract & 24 \\
Introduction & 422 \\
Sentence & \\
& Metabolic entities count \\
Entities type & $747(14.09,12.69)^{*}$ \\
Metabolite & $675(12.74,11.55)^{*}$ \\
GP & $1422(26.83,21.73)^{*}$ \\
Total & \\
Events type & \\
Metabolic production & $99(1.87,2.18)^{*}$ \\
Metabolic consumption & $45(0.85,1.52)^{*}$ \\
Metabolic reaction & $62(1.17,2.03)^{*}$ \\
Positive regulation & $34(1.17,2.03)^{*}$ \\
Total & $240(4.53,4.74)^{*}$ \\
\hline
\end{tabular}

3

4

*The average number of units and per abstract and Standard deviation (SD). ${ }^{*}$ The average number of arguments per metabolic event.

$115(0.42,0.99)^{*},\left(1.10^{\#}\right)$

$132(0.49,1.07)^{*},\left(1.22^{\#}\right)$

$134(0.49,0.94)^{*},\left(2.12^{\#}\right)$

$99(0.36,0.65)^{*},\left(1.76^{\#}\right)$

$480(1.77,2.35)^{*},\left(1.51^{\#}\right)$

\section{Test corpus}

Entities type 


\section{Table 5 (on next page)}

Table 5 - Performance of MEE module on metabolic entities corpus using five-fold crossvalidation 
1 Table 5 - Performance of MEE module on metabolic entities corpus using five-fold 2 cross-validation

\begin{tabular}{llll}
\hline Events type & Recall (\%) & Precision (\%) & F-score (\%) \\
\hline Metabolic production & 62.94 & 55.79 & 59.15 \\
Metabolic consumption & 41.67 & 58.27 & 48.59 \\
Metabolic reaction & 24.50 & 33.56 & 28.32 \\
Positive regulation & 30.70 & 45.57 & 36.69 \\
Micro average & 38.16 & 48.18 & 42.59 \\
\hline
\end{tabular}

3 
Table 6(on next page)

Table 6 - Performance of MEE module on test corpus for tagging GP and metabolite 
1 Table 6 - Performance of MEE module on test corpus for tagging GP and metabolite

\begin{tabular}{lll}
\hline & \multicolumn{1}{c}{ GP entities } & Metabolite entities \\
\hline \multicolumn{2}{l}{ Superpathway of leucine, valine, and isoleucine biosynthesis } \\
\hline Recall (\%) & $81.79(274 / 335)^{*}$ & $85.51(301 / 352)^{*}$ \\
Precision (\%) & $92.88(274 / 295)^{\#}$ & $91.77(301 / 328)^{\#}$ \\
F-score (\%) & 86.98 & 88.53 \\
\hline \multicolumn{2}{l}{ Superpathway of pyridoxal 5'-phosphate biosynthesis and salvage } \\
\hline Recall (\%) & $84.62(297 / 351)^{*}$ & $65.16(245 / 356)^{*}$ \\
Precision (\%) & $83.90(297 / 354)^{\#}$ & $87.81(245 / 279)^{\#}$ \\
F-score (\%) & 84.26 & 74.81 \\
\hline
\end{tabular}

2

$3{ }^{*}$ The number in parenthesis indicates correctly predicted entities/number of total correct entities.

4 \# The number in parenthesis indicates correctly predicted entities/number of total predicted entities.

5 Note: Performance of MEE module on test corpus for tagging GP and metabolite used the trained model from five 6 fold cross-validation (see Table 5). 
Table 7 (on next page)

Table 7 - Performance of MINR module on test corpus 


\section{Table 7 - Performance of MINR module on test corpus}

\section{Enzyme-metabolite interaction}

\begin{tabular}{lc}
\hline${ }^{a}$ Superpathway of leucine, valine, and isoleucine biosynthesis \\
\hline Recall (\%) & $64.65(64 / 99)^{*}$ \\
Precision (\%) & $84.21(64 / 76)^{\#}$ \\
F-score (\%) & 73.14 \\
\hline${ }^{b}$ Superpathway of pyridoxal 5'-phosphate biosynthesis and salvage \\
\hline Recall (\%) & $76.84(73 / 95)^{*}$ \\
Precision (\%) & $90.12(73 / 81)^{\#}$ \\
F-score (\%) & 82.95 \\
\hline
\end{tabular}

2

3 a Under the Superpathway of leucine, valine, and isoleucine biosynthesis, 88 identified enzyme-metabolite 4 interactions were found by manual curation and used as a reference for performance evaluation.

5 b Under the Superpathway of pyridoxal 5'-phosphate biosynthesis and salvage, 87 identified enzyme-metabolite 6 interactions were found by manual curation and used as a reference for performance evaluation.

$7 \quad{ }^{*}$ The number in parenthesis indicates correctly predicted entities/number of total correct entities.

8 \# The number in parenthesis indicates correctly predicted entities/number of total predicted entities.

9 Note: Performance of MINR module on test corpus used the trained model from five fold cross-validation (see 10 Table 5).

11

12 


\section{Table 8 (on next page)}

Table 8 - The reconstructed metabolic interaction network using ME corpus and EcoCyc extraction applications 
1 Table 8 - The reconstructed metabolic interaction network using ME corpus and EcoCyc 2 extraction applications

\begin{tabular}{lllllll}
\hline Entities & \multicolumn{2}{l}{$\begin{array}{l}\text { EcoCyc extraction } \\
(\mathbf{2 , 3 7 3} \text { abstracts and titles) }\end{array}$} & \multicolumn{2}{l}{ ME corpus (271 abstracts and titles) } \\
& $\begin{array}{l}\text { \# Correctly } \\
\text { predicted }\end{array}$ & $\begin{array}{l}\text { \# Total } \\
\text { predicted }\end{array}$ & $\begin{array}{l}\text { Precision } \\
(\%)\end{array}$ & $\begin{array}{l}\text { \# Correctly } \\
\text { predicted }\end{array}$ & $\begin{array}{l}\text { \# Total } \\
\text { predicted }\end{array}$ & $\begin{array}{l}\text { Precision } \\
(\%)\end{array}$ \\
\hline Enzyme & 193 & 293 & 69.93 & 58 & 74 & 78.38 \\
$\begin{array}{l}\text { Metabolite } \\
\text { Enzyme- }\end{array}$ & 190 & 260 & 70.63 & 80 & 113 & 70.80 \\
$\begin{array}{l}\text { metabolite } \\
\text { interaction }\end{array}$ & 234 & 501 & 46.71 & 76 & 137 & 55.47 \\
\hline
\end{tabular}

3 Note: ME corpus and EcoCyc extraction was done using the same training dataset (i.e., ME corpus for 271 abstracts 4 and titles). To reconstruct the metabolic interaction network, E. coli K-12 MG1665 (iJO1366) genome-scale 5 metabolic network [45] was used as an interaction reference. 


\section{Figure 1 (on next page)}

Figure 1 - A schematic diagram outlining the development steps for the proposed integrated TM framework.

Note: Metabolic entities corpus size means the effect of different corpus sizes on performance of MEE module within the integrated TM framework. 
Construction of Metabolic Construction of test corpus Entities (ME) corpus
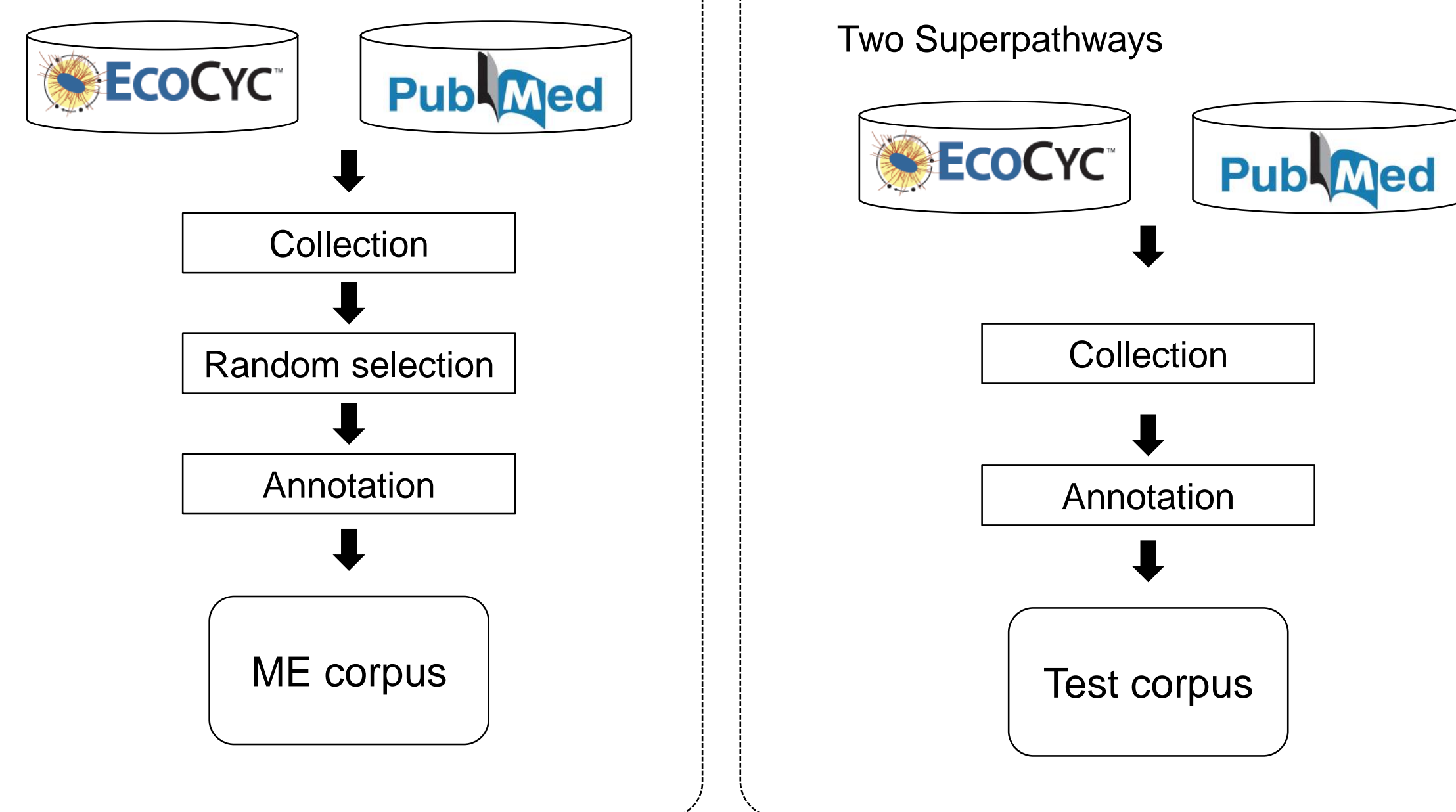

$\downarrow$

\section{Development of Integrated TM framework}

\section{Development of Metabolic Development of Metabolic} Event Extraction

(MEE) module Interaction Network
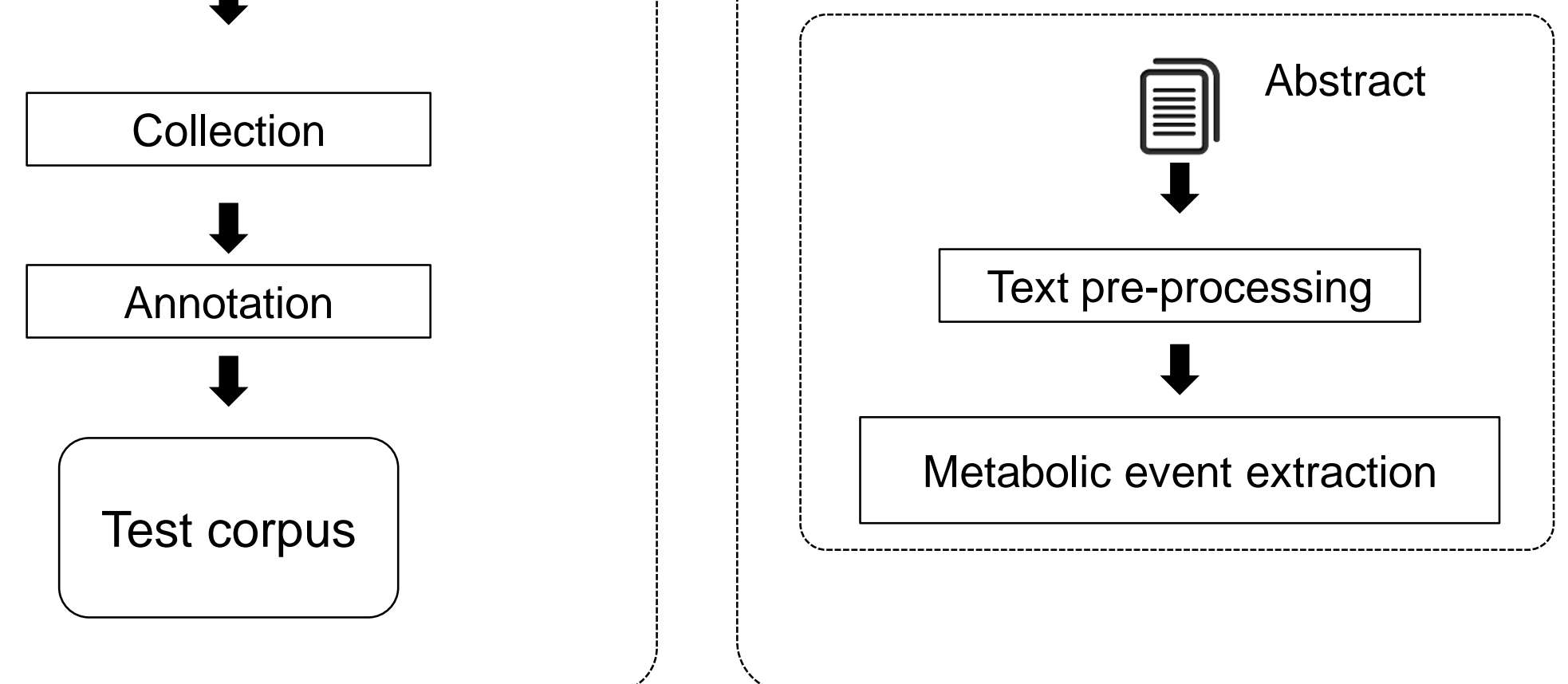

Reconstruction (MINR) module

Mapping from metabolic event to metabolic interaction $\downarrow$

Reconstructing metabolic interaction network

\section{Evaluation of Integrated TM framework}

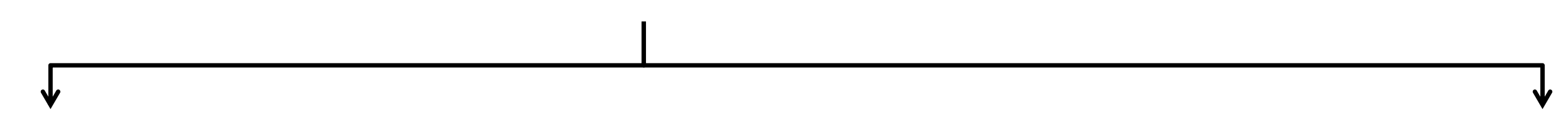

MEE module

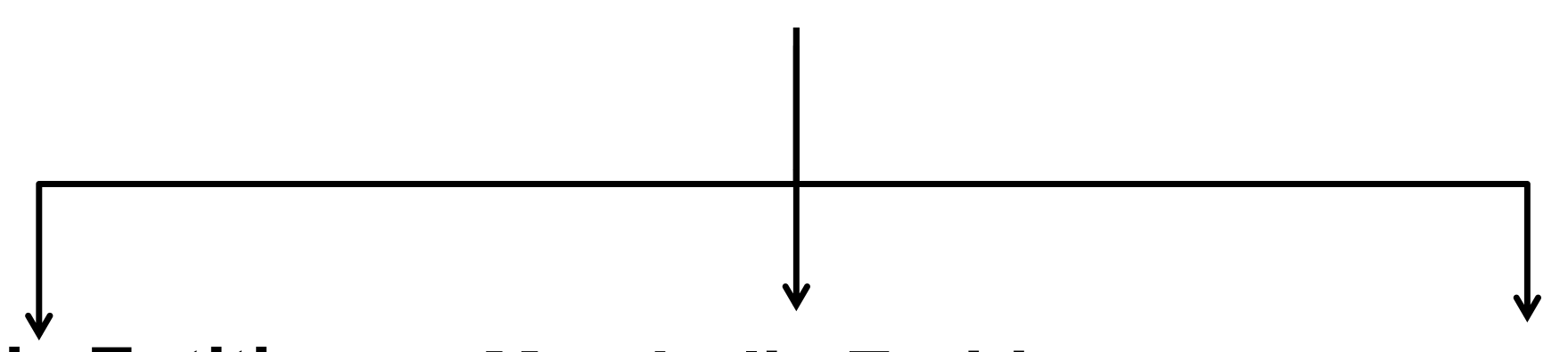

Metabolic Entities

(ME) corpus
Metabolic Entities

(ME) corpus size
Test corpus

\section{MINR module}

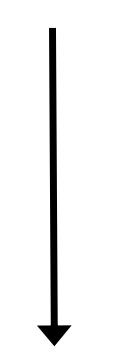

Test corpus

\section{Application of integrated TM framework}


Figure 2 (on next page)

Figure 2 - A schematic annotation of metabolic entities and events

(A) Hierarchical representation of metabolic entities and events. (B) An example of metabolic entities and events annotation. 
(A)

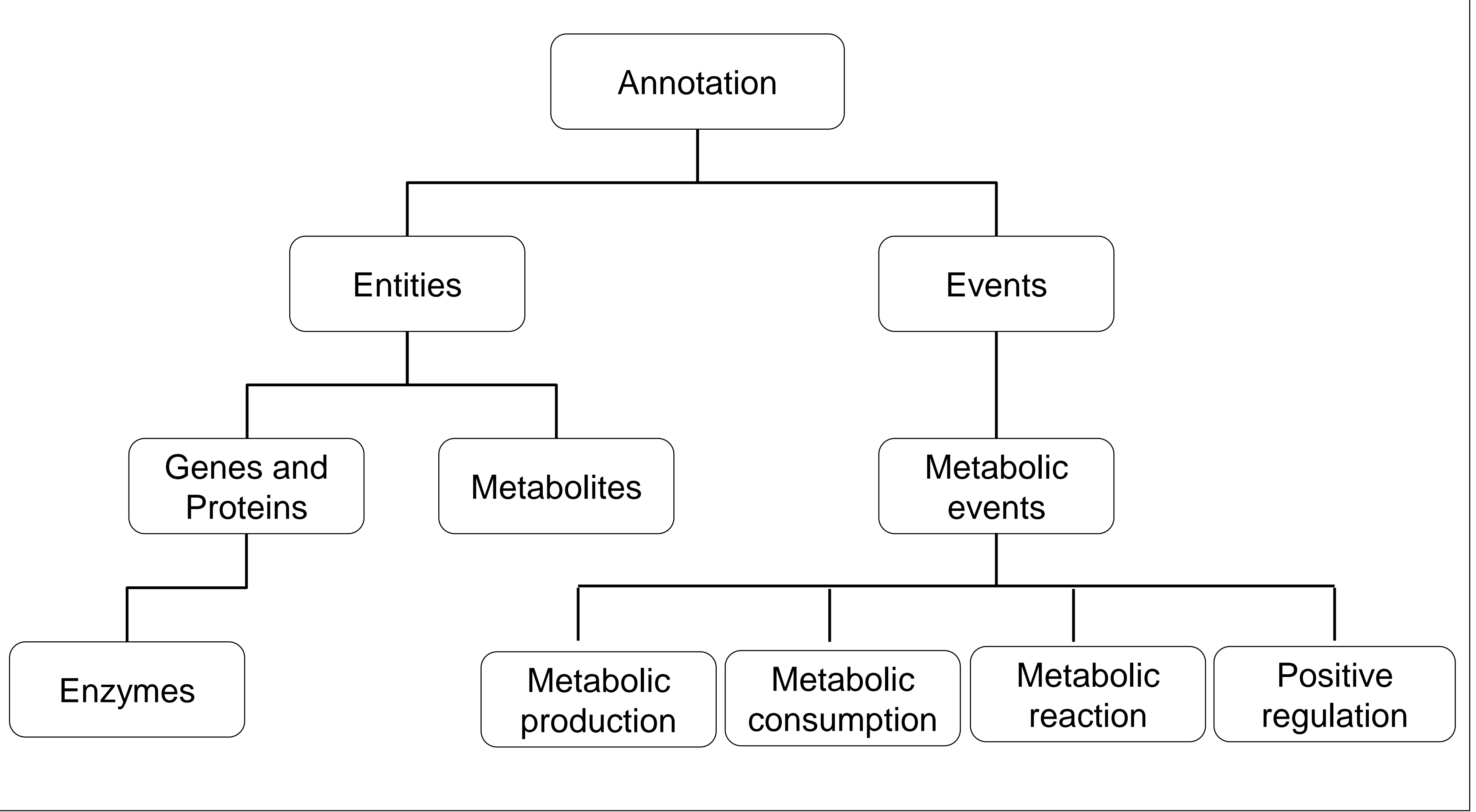

(B)

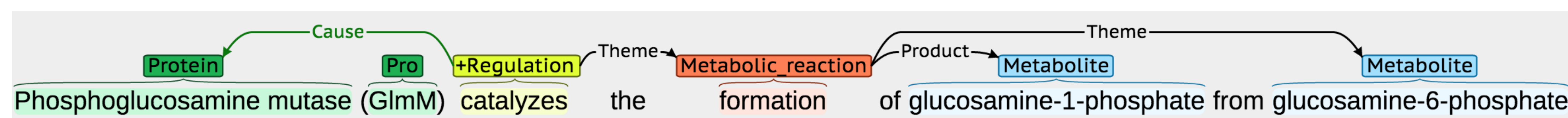


Figure 3 (on next page)

Figure 3 - An illustration showing the process implementation of the proposed integrated TM framework.

(A) Metabolic Event Extraction (MEE) module; (a1) Input text for MEE module, (a2) Output text from MEE module, (a3) Text output visualization using BRAT [33]. (B) Metabolic Interaction Network Reconstruction (MINR) module; (b1) Intermediate graphical output from MINR module, (b2) Graphical output from MINR module, (b3) enzyme-metabolite interactions pair from MINR module. 


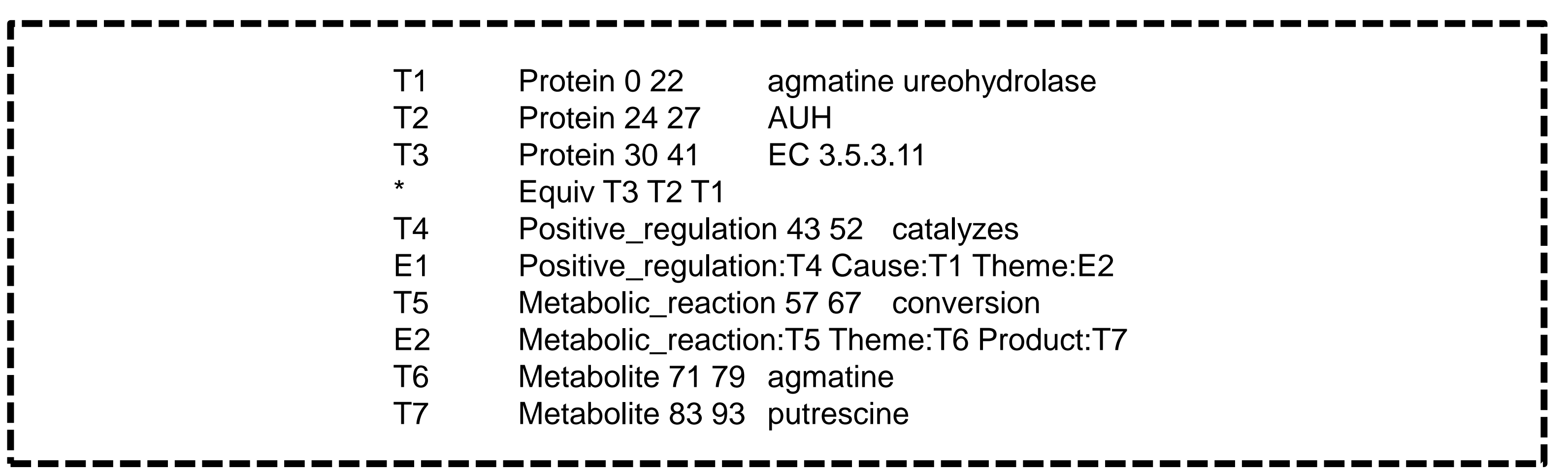

(a3)

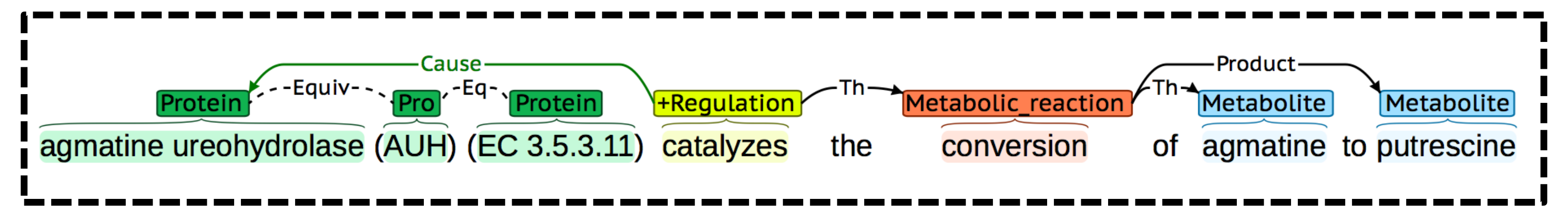

(B)

(b1)

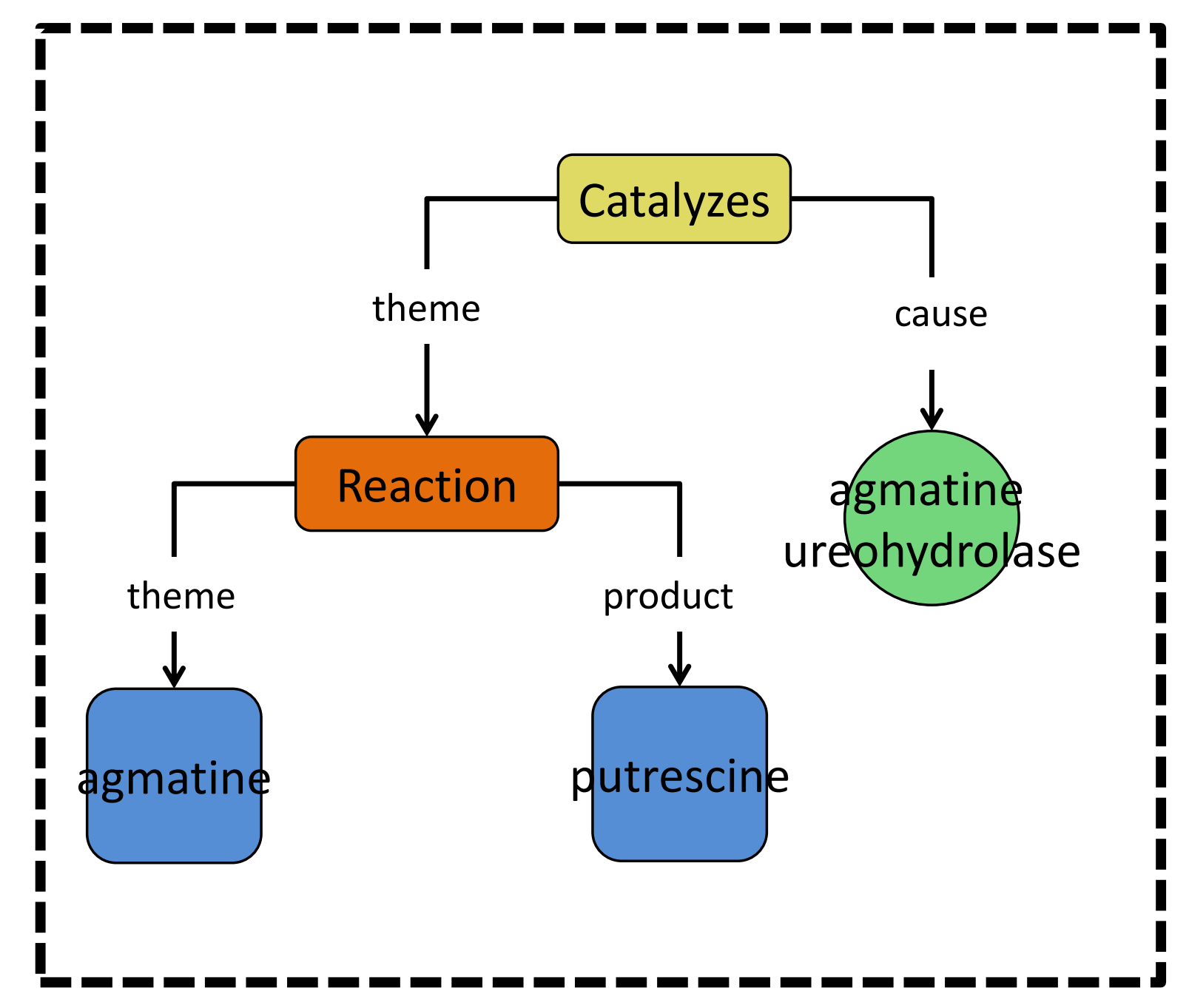

(b2)

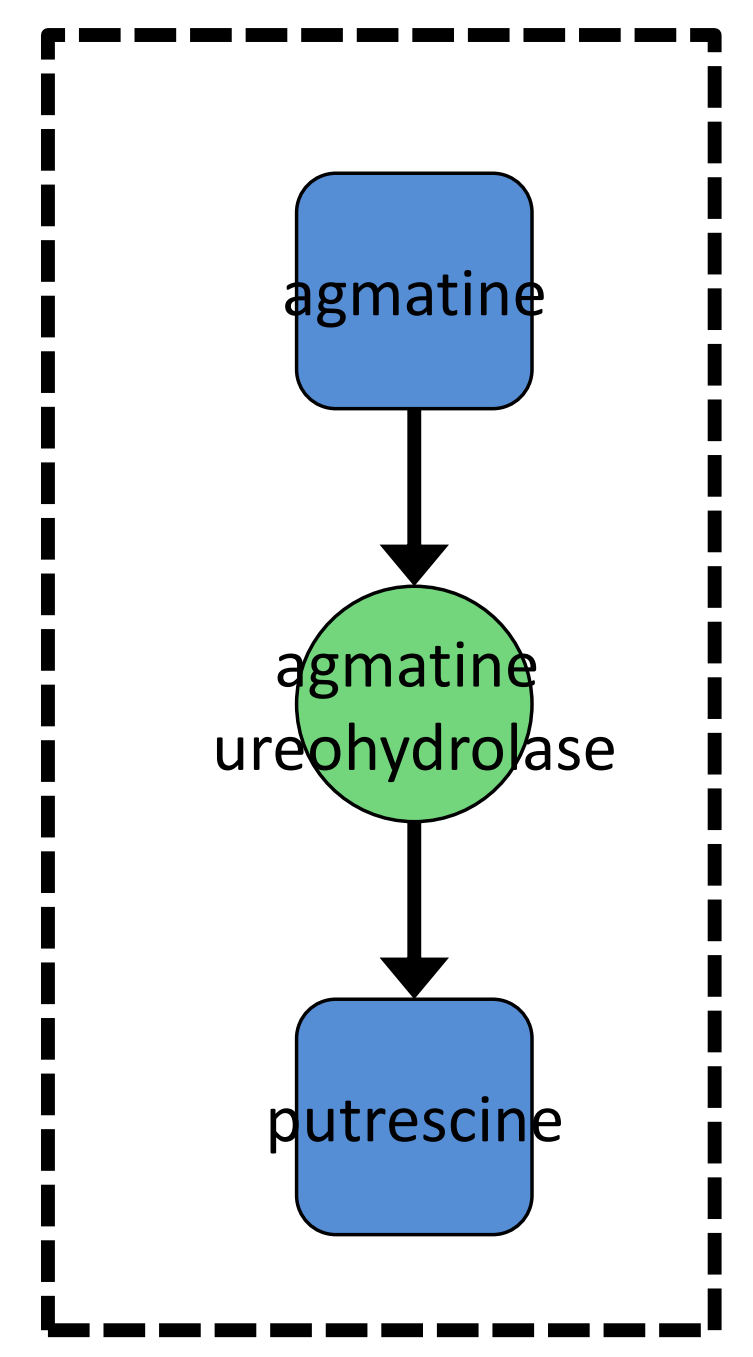

(b3)

Enzyme: agmatine ureohydrolase
Metabolite: agmatine
Enzyme: agmatine ureohydrolase
Metabolite: putrescine


Figure 4 (on next page)

Figure 4 - Learning curves showing performance of the Metabolic Event Extraction (MEE) module on the different corpus sizes using five-fold cross-validation.

Each of metabolic event types and total are plotted against (A) Recall, (B) Precision, and (C) F-score. The total was calculated by summation of the individual true positives, false positives, and false negatives for each of metabolic event types. 


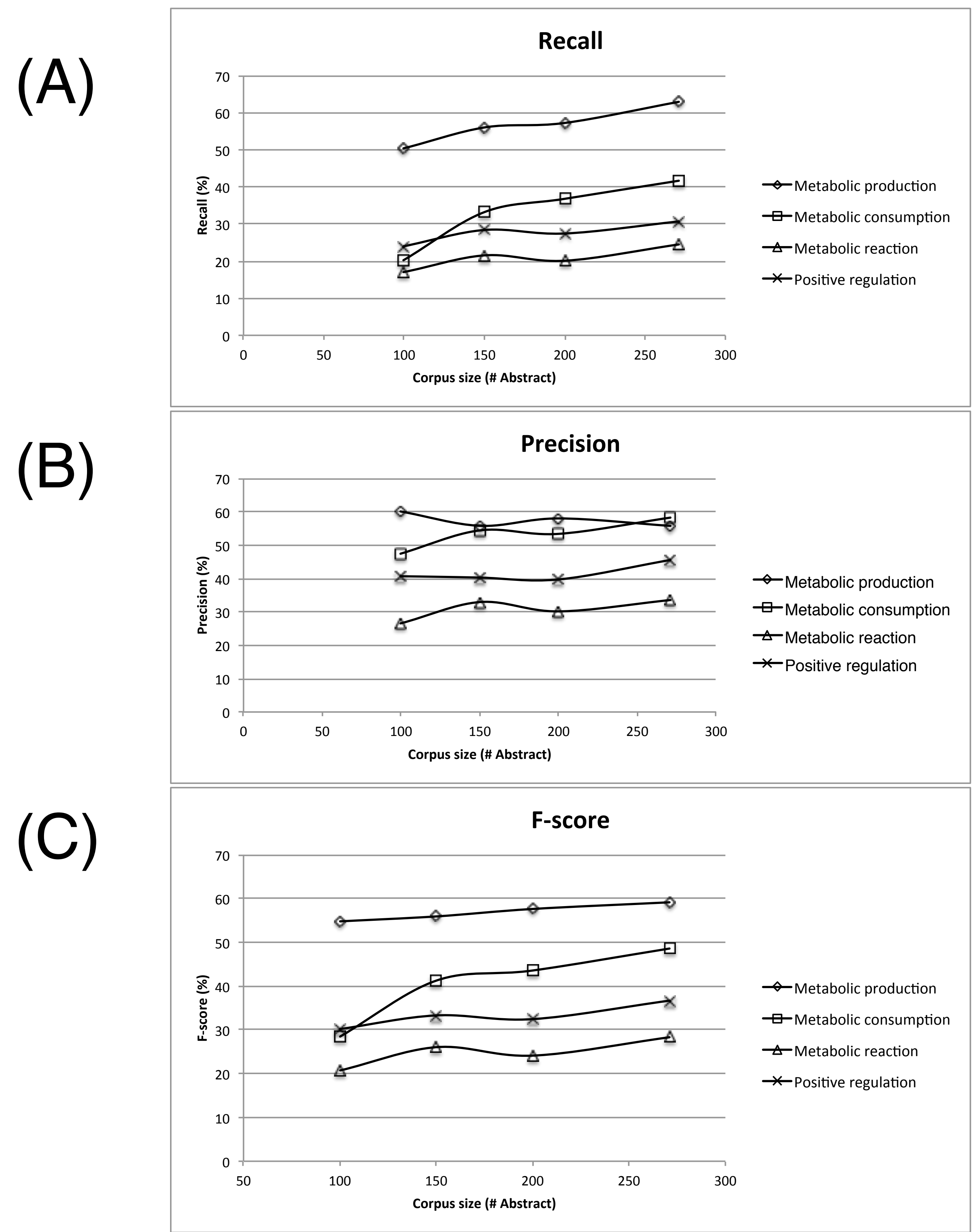




\section{Figure $\mathbf{5}$ (on next page)}

Figure 5 - Comparative analysis of two TM systems on test corpus for the Superpathway reconstruction of leucine, valine, and isoleucine biosynthesis.

(A) Our integrated TM framework (MEE and MINR modules), (B) TM system developed by Czarnecki et al. [23]. The extracted interactions were overlaid to the reference metabolic pathway from the EcoCyc database. A grey node means correct extraction. A white node means incorrect extraction. An edge with an arrow means correct extraction. An edge with a dash arrow means incorrect extraction. This bipartite metabolic graph is created using Cytoscape version 3.0.1. *The extracted enzyme-metabolite interaction indicates the number of binary interaction. \#The number in parenthesis is a percentage of extracted mentions/events in comparison to the reference metabolic pathway from the EcoCyc database. 

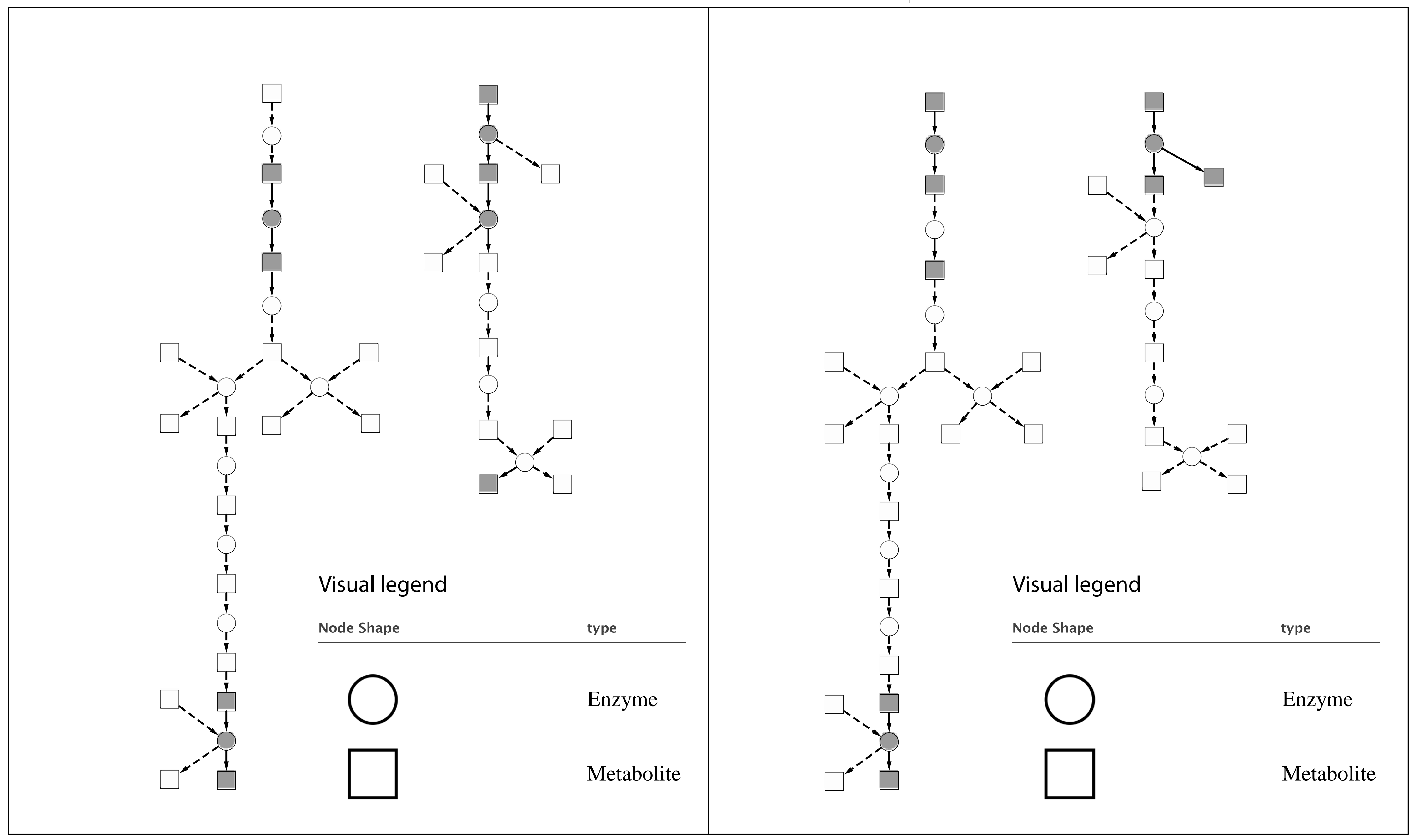

\begin{tabular}{|l|l|}
\hline \multicolumn{2}{|l|}{ (A) Our integrated TM framework } \\
\hline $\begin{array}{l}\text { Enzymes and } \\
\text { metabolites }\end{array}$ & $11(26 \%)^{\#}$ \\
\hline $\begin{array}{l}\text { Enzyme-metabolite } \\
\text { interactions* }\end{array}$ & $10(25 \%)^{\#}$ \\
\hline
\end{tabular}

(B) TM system developed by Czarnecki et al. [23]

\begin{tabular}{|l|l|}
\hline $\begin{array}{l}\text { Enzymes and } \\
\text { metabolites }\end{array}$ & $11(26 \%)^{\#}$ \\
\hline $\begin{array}{l}\text { Enzyme-metabolite } \\
\text { interactions* }\end{array}$ & $8(20 \%)^{\#}$ \\
\hline
\end{tabular}


Figure 6 (on next page)

Figure 6 - The integrated TM framework application. 

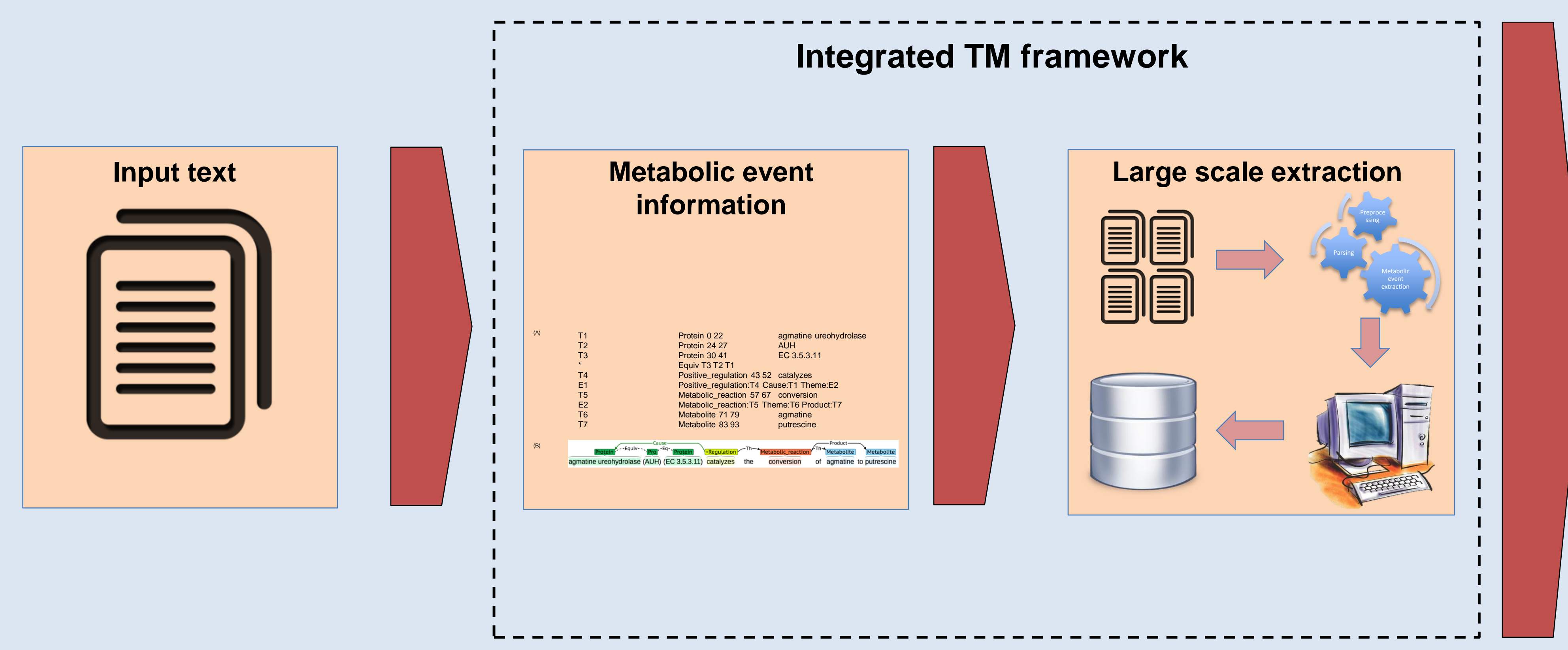

Metabolic interaction

database and network
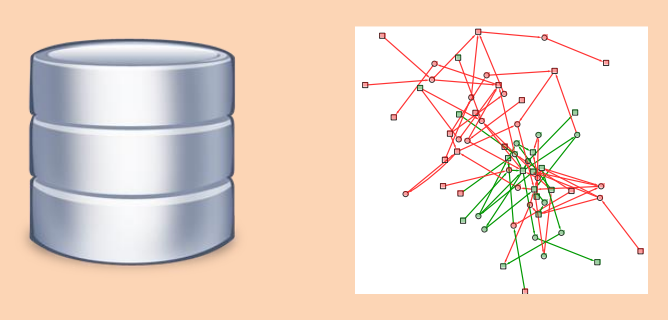

Knowledge discovery

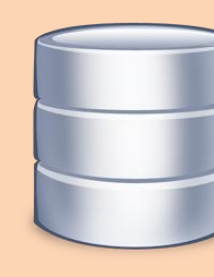

